\title{
Vida Cotidiana en un Poblado de la EdAd del Hierro. Cerro de los encaños, Villar del Horno (cuenca).
}

\author{
Everyday life in a settlement of the Iron Age. \\ Cerro de los Encaños, Villar del Horno (Cuenca)
}

ADRIANo GómEZ RuíZ

Recibido: 07/11/2017

Revisado: 11/03/2019
Aceptado: 24/05/2019

Publicado: 05/07/2019
RESUMEN

El presente trabajo es el resultado del estudio, exploración y elevación del plano de un Conjunto Ibérico entre los siglos VII y III a. C. asentado en el curso alto del río Cigüela, afluente del río Guadiana.

Los habitantes de la zona renunciaron a las ventajas que ofrecen una gran concentración humana (como las cercanas ciudades de Segóbriga, Valeria o Ercávica) para extenderse a lo largo del valle.

El sitio arqueológico es una colina exenta en el centro del valle, de 20 metros de altura en el momento y con algo más de una hectárea.

La excavación proporcionó dos momentos de ocupación, Villar I, es un pueblo de la primera Edad del Hierro, con alfarería hecha a mano de la tradición de la Edad del Bronce, y Villar II, perteneciente a la segunda Edad del Hierro.

\section{Abstract}

The present work is the result of the study, exploration and raising plane of an Iberian settlement between the VIIth and IIIth century B.C. seated in the high course of the Cigüela river, tributary of the Guadiana river.

The inhabitants of the zone resigned the advantages that there provide a great human concentration (such as the nearby cities of Segóbriga, Valeria or Ercávica) to be spread along the valley.

The archaeological site is an exempt hill in at the centre of valley, of 20 metres altitude at the momento and with some smaller than one hectare.

The excavation provided two moments of settlements, Villar I, is a village of the first Iron Age, with pottery made by hand of tradition of the Bronze Age, and Villar II, belonging to the second Iron age.
Palabras Clave

Edad del Hierro; río Ciguela; Cuenca; cerámica; asentamiento.

\section{KEY WORDS}

Iron age; Cigüela river; Cuenca; pottery; settlement.

adriano1943_grz@hotmail.es 


\section{INTRODUCCIÓN}

Expondremos en estas líneas cómo podría haber sido la vida cotidiana en un pequeño poblado entre los siglos VI y IV a.C Para ello nos basaremos en las campañas de excavaciones desarrolladas en el yacimiento llamado Cerro de los Encaños y, sobre todo, en el material arqueológico proporcionado por dichas excavaciones y la interpretación que damos a ese material.

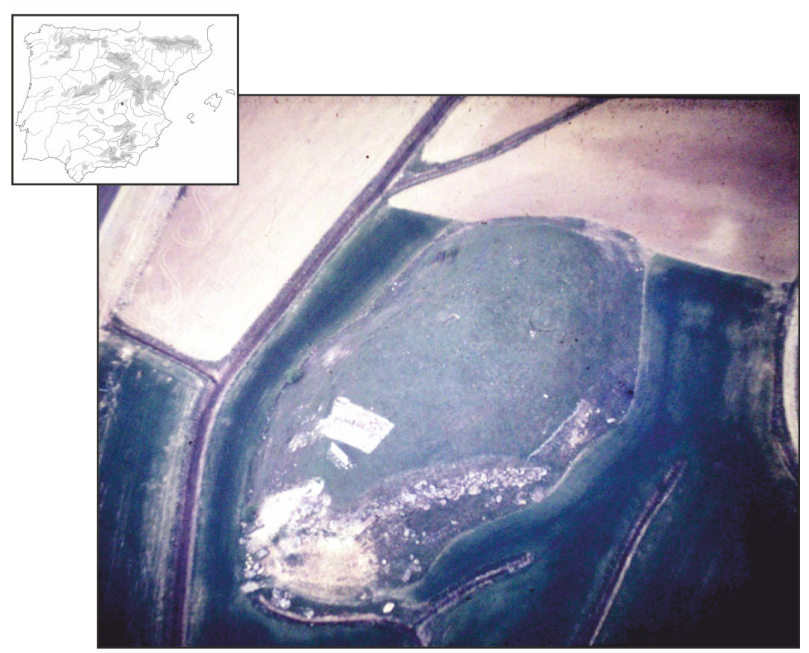

Figura 1.- Localización y Vista aérea del yacimiento.

El Cerro de los Encaños, en Villar del Horno (Cuenca), se halla situado en el valle del curso alto del río Cigüela. Es un yacimiento arqueológico con singulares características para el estudio de la Edad del Hierro en la provincia de Cuenca. Su peculiar orografía, su situación geográfica y su definitivo abandono sin que otras poblaciones lo ocuparan en intensidad, nos ofrecen ahora un lugar idóneo para el análisis de una oscura época dentro de la Protohistoria peninsular.

Ubicado sobre un cerro testigo, un pequeño promontorio exento en el centro del valle, de unos 20 metros de altura en la actualidad y con una extensión habitable de poco más de media hectárea, es indudable que los primeros pobladores eligieron el lugar óptimo para desarrollar una actividad agropecuaria en el valle y cobijarse en el montículo ante cualquier manifestación hostil foránea (Ortega y del Valle, 2004).

Sus características geográficas nos hablan de una pequeña población estable que explota el amplio y fértil valle, interrelacionada con pequeños grupos próximos y afines y que, en común, organizan la defensa de la zona en momentos de peligro(Valiente Cánovas, 1982). Ocupa el lugar más apropiado para dominar la entrada de una amplia zona cultivable, atisbar un posible peligro con la suficiente antelación para la defensa o avistar caravanas de gentes foráneas con quienes intercambiar la materia prima por objetos y utensilios de "lujo" y vasijas decoradas fabricadas a torno (Valiente Cánovas,1981).

La vida cotidiana en unos momentos de gran inestabilidad fue posible gracias a la defensa organizada de pequeños pero numerosos poblados que, dominando extensas zonas desde su altitud, podían comunicarse entre sí y preparar con suficiente antelación el ataque o la defensa (Gómez Ruiz, 1999, 83-87).

El inconveniente del reducido espacio habitable del Cerro de los Encaños quedó salvado por la estructura social, ya que estas gentes no tendieron a una gran concentración humana en la zona, sino por el contrario, a diseminarse en pequeños poblados situados en estratégicos montículos, fuertemente defendidos, en el valle del Cigüela y en los rebordes circundantes (Ruiz y Álvarez, 2015).

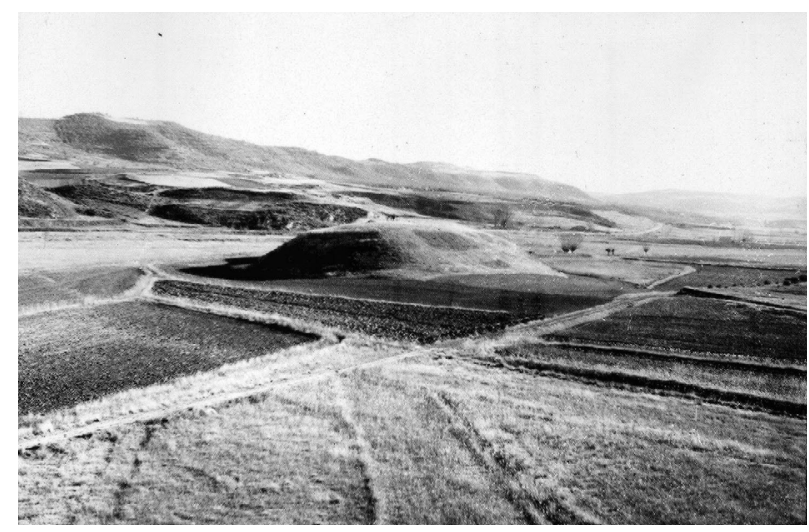

Figura 2.- El Cerro de los Encaños, en el centro de la foto, visto desde el Oeste

Las excavaciones realizadas en el cerro han puesto al descubierto dos momentos ocupacionales: Villar I es un poblado de la Primera Edad del Hierro, con cerámica a mano de tradición del Bronce, tanto lisa como decorada. Algún que otro fragmento de cerámica pintada con rombos rojo oscuro, tipo hallsttático, nos identifica a estas gentes (o por lo menos se relacionan) con el mundo centroeuropeo tras su penetración pirenaica y su expansión por el valle del Ebro. Esta cerámica pintada comienza a ser relativamente abundante en la provincia de 
Cuenca tras los últimos descubrimientos (Almagro Gorbea, 1969).

La decoración no pintada de estos vasos a mano, vasijas, cuencos y platos presentan una gran variedad de motivos, todos ellos vinculados al mundo de la Primera Edad del Hierro (Almagro Gorbea, 1988), desde ungulaciones en el borde, reticulados, incisiones finas, acanaladuras, incisiones en zig-zag, onduladas, aspeadas, rehundimientos digitales y un largo etcétera. Una gran parte de estas vasijas son decoradas con otra técnica diferente, añadiendo elementos a la superficie, como mamelones perforados o sin perforar, de pastillas, mamelones rehundidos, botones cerámicos, gallones verticales, etc. (Urbina Martínez, 2015).

Los objetos de hierro están escasamente representados en este primer poblado, aunque no debemos olvidar que se ha excavado una reducida superficie de este nivel: unos 35 metros cuadrados.

La cerámica a torno es abundante porcentualmente (casi el $40 \%$ ), aunque hemos de pensar que al inicio del asentamiento (quizá al final del siglo VIII o primera mitad del siglo VII) no se conoció; posteriormente, llegó como producto de importación coexistiendo con la cerámica a mano autóctona hasta la destrucción de este primer poblado. Hemos hallado un solo nivel de habitaciones en Villar I, por lo que su larga pervivencia pudo originar la existencia de estas cerámicas a torno, pintadas o no, compitiendo con la cerámica a mano, típicamente del Hierro I.

En la segunda mitad del siglo VI fue violentamente destruido, volviéndose a ocupar tras un lapsus de tiempo suficientemente largo para que Villar I quedara "sellado" por una capa de tierra sobre la que se construyó un nuevo poblado que hemos llamado Villar II. Este nuevo asentamiento está formado por gentes afines a los primeros; siguen fabricando cerámica a mano con los mismos elementos decorativos (si exceptuamos la cerámica pintada hallsttática, tipo Carrascosa), pero la cerámica a torno es mucho más abundante y diversificada, aunque ésta hay que incluirla en un "horizonte ibérico antiguo", tanto por los motivos decorativos como por las pinturas y las pastas (Martín Valls,1997).

Villar II continúa con las actividades económicas precedentes; explotando el valle y desarrollando una ganadería que se hace patente en el análisis de los restos de animales aparecidos. Se han realizado dos de estos análisis; el primero, por el Dr. Morales Muñiz del Departamento de Zoología y Fisiología animal de la Facultad de Ciencias de la Universidad Autónoma de Madrid (Gómez Ruíz, 1986, 265350), y el segundo, realizado por la Dra. Fernández Sánchez, adscrita a la Estación Biológica de Doñana del Consejo Superior de Investigaciones Científicas. Ambos estudios coinciden en que la cabaña ganadera de Villar II está representada por caballos, asnos, bovinos, cabras, ovejas y cerdos; siendo el perro el animal doméstico auxiliar, tanto en las labores ganaderas como cinegéticas. El Cerro de la Mesa (Toledo), presenta las mismas especies faunísticas (Ortega y del Valle, 2004).

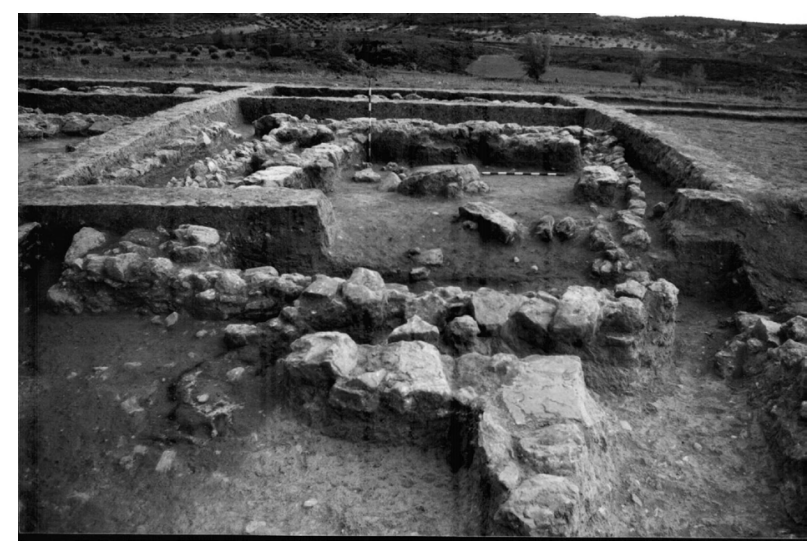

Figura 3-. Recinto 21 de Villar II. Edificio rectangular con un hogar central.

La caza fue un complemento importante en los hábitos alimentarios de esta población; están representados el ciervo y el jabalí, cuyos colmillos y astas se utilizaron para desarrollar una artesanía del hueso (Valiente Cánovas, 1986).

También la depredación fue otra actividad económica de los habitantes del poblado, representada en la recolección de caracoles acuáticos y por la presencia de cáscaras de huevos de aves.

\section{CONSTRUCCIONES}

Sospechamos que toda la cima del cerro estuvo ocupada por construcciones de dos tipos: la morada propiamente dicha y el establo para el ganado, construido éste con tapiales sujetos con losas colocadas encima. Se han localizado no menos de 22 recintos entre todas las campañas y muchos vestigios de otros destruidos, pero calculamos un número de habitaciones que puede aproximarse a las 70, si excluimos los establos para el ganado.

La cabaña se construía con muros de piedra de regular tamaño unidas con barro, hasta una altura 
aproximada de 1'20 m. Estos muros, con una anchura de 50-60 cm, se remataban con losas, a partir de las cuales se elevaba la pared con adobes. Esta forma constructiva se debía a la carencia de cimientos que haría peligrar la verticalidad de un muro elevado, ya que la base sólo se sujetaba con algunas losas verticales por el exterior; por el interior se enfoscaría con arcilla alisada formando un enlucido uniforme cuyos restos se hallan caídos sobre el pavimento con relativa frecuencia (Contreras Martínez et al.,2014)

La mayoría de las cabañas excavadas presentan plantas cuadrangulares y rectangulares, aunque también existen plantas poligonales y romboidales, estas últimas, sobre todo, levantadas en el inicio de la ladera para adaptarse mejor a la curva de nivel (Rodríguez Hernández, 2017). Tenemos paralelos de casas romboidales en el poblado de La Hoya (Álava).

La techumbre, posiblemente a dos aguas, era de ramas y barro soportada por vigas de madera trasversales. Ello queda demostrado por el grosor de la capa de carbón y cenizas que se ha depositado sobre los pavimentos tras el incendio del poblado. Cuando la habitación presenta una dimensión mayor de lo corriente, se hacen necesarios los postes verticales hincados en el suelo y acuñados por losas.

Algunas viviendas presentan un banco de piedra y barro adosado a uno de los muros (Ortega y del Valle, 2004). Existen paralelos de estos bancos adosados en varios yacimientos de la época; concretamente nos vamos a referir a los poblados de Raso de Candeleda en Ávila, La Hoya en Álava y Alto de la Cruz en Cortes de Navarra, que tantas similitudes presenta con el Cerro de los Encaños a pesar de la separación geográfica, pero también hemos de referirnos para documentar este hecho a aquel pasaje de Estrabón que, refiriéndose a galaicos y astures, dice: “...comen sentados sobre bancos construidos alrededor de las paredes, alineándose en ellos según sus edades y dignidades..." (García Bellido,1968).

Los pavimentos de estas casas de realizaban con cuidada técnica a base de una capa de pequeños cantos rodados (quizá con la doble finalidad de allanamiento y drenaje), una segunda capa de fragmentos de cerámica y todo unido y mezclado con una espesa masa de barro arcilloso cuya superficie se alisaba con esmero y se endurecía, quizá con fuego, antes de ocupar la vivienda.

Algo imprescindible en estas cabañas era el hogar, realizado con la misma técnica que el pavimento, pero, generalmente, situado a más elevación que

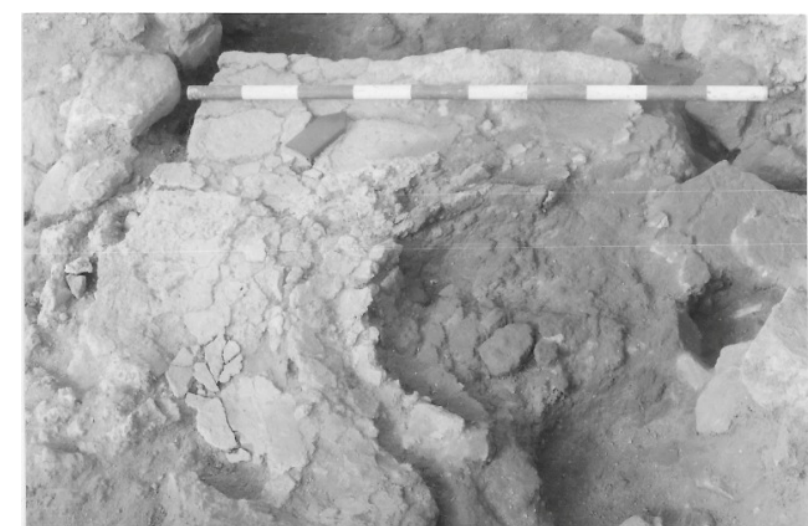

Figura 4.- Horno abovedado sobre una plataforma cuadrangular delimitada por adobes.

éste y rodeado algunas veces por piedras de regular tamaño o hileras de adobes. No ocupaba un lugar uniforme en todos los recintos, sino que unas veces se halla adosado a un muro, otras, en una esquina y algunas, en el centro de la cabaña. Por lógica, su existencia nos habla de la parte principal de la vivienda, y su ausencia nos indica que el recinto es anexo o secundario. Aparecen hogares de las mismas características que los del Cerro de los Encaños en yacimientos tan alejados geográficamente como Reillo (Cuenca), La Coronilla (Guadalajara), Puig de Castellet en Lloret del Mar (Gerona), El Raso de Candeleda en Ávila (Fernández Gómez, 2001), Alto de la Cruz en Navarra (Maluquer de Motes, 1954), y un largo etcétera (Ortega y del Valle, 2004)

Distintos a los hogares, pero quizá con doble función, se hallan los hornos de cocer abovedados, en forma de colmena y realizados con arcilla, posiblemente, para la fabricación del pan. Hornos de estas características como los que se han descubierto en el Cerro de los Encaños, son frecuentes en los poblados de la época (Cabello Caja et. al., 1992)

Los recintos excavados son todos de pequeñas dimensiones (contrastando con el grosor de los muros), entre 25 y 35 metros cuadrados, aunque muchos de ellos pueden ser anexos de una unidad familiar. Rara vez las casas están adosadas, sino que presentan muros paralelos. Pocas veces se aprovecha en común una pared para dos cabañas, quedando un reducido espacio entre ellas, quizá con vista al desagüe de las techumbres.

Además de estos recovecos entre cabañas de difícil tránsito, el poblado presenta calles longitudinales y rectilíneas de una anchura que oscila entre 2'50 m y 2'60 m, y que facilitan el acceso a las vi- 

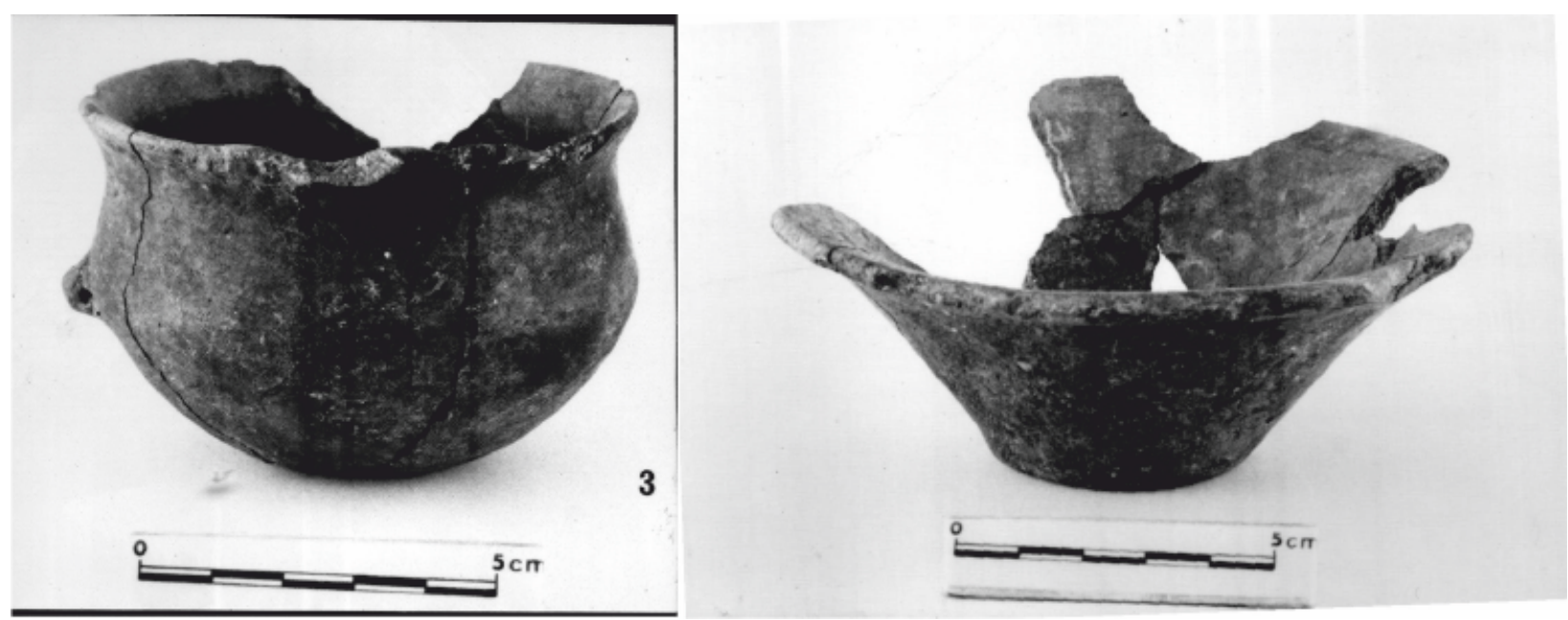

Figura 5.- Cerámicas fabricadas a mano procedentes de Villar-I.

viendas y establos. No se ha hallado ningún tipo de pavimentación en estas calles, pero sí se nota un preconcebido plan urbanístico en el poblado con sus recintos alineados.

No hay vestigios de ventanas, quizá porque no existieran y la única claridad en el interior procedía de la puerta de entrada, o porque estas aberturas se situaban por encima del muro de piedras; es decir, en la pared superior de tapial o adobes, que no se conserva (Ortega y del Valle, 2004).

\section{DEFENSA}

El Cerro de los Encaños, como un cerro testigo en el valle del río Cigüela, se presenta como un bastión natural apto para albergar a una pequeña población que se siente segura en su cima. Su actual altitud, de unos $20 \mathrm{~m}$, es inferior a la de la época de ocupación, pues el aporte aluvial del río ha elevado el valle en unos dos metros. No obstante, esta elevación no ofrecía suficiente defensa, por lo que se rodeó la cima del cerro con una gruesa muralla de 1'50 m de anchura. Esta defensa ha desaparecido a lo largo del tiempo por el aprovechamiento de sus piedras y, más recientemente, para la construcción de una pista próxima al cerro; pero aún quedan vestigios de su base a dos metros por debajo de las últimas construcciones. Los poblados del Bronce y Primera Edad del Hierro se presentan poco fortificados; pero en le Segunda Edad del Hierro se fortifican más eficazmente, pues como recoge Jesús Rodríguez Hernández en su tesis doctoral “...En eta segunda etapa las murallas rodean por completo las aldeas, en ocasiones mediante varios recintos (Rodríguez Hernández, 2017) Aparecen los torreones circulares como en castillejo II (Lámina 6) o en el Cerro de la Virgen de la Cuesta en Alconchel de la Estrella en Cuenca (Aguado Millán, 2018). También encontramos este tipo de fortificación en lugares tan alejados como en La Coraja de Aldeacentena en Cáceres (Ortega et al., 2004).

$\mathrm{El}$ acceso al poblado se realizaba por una rampa artificial y rectilínea construida en la ladera $S$ del cerro. Aunque no quedan restos visibles algunos, suponemos (y futuras excavaciones lo podrán demostrar) que dos fuertes bastiones flanqueaban la entrada en la parte superior de la rampa, por ser el lugar más vulnerable (Gras et al., 1984).

En la actualidad, el acceso al yacimiento no presenta dificultades gracias a la canalización del Cigüela y un riachuelo afluente que rodean el cerro, pero en la época en que fue elegido para asentamiento, la zona circundante se presentaba como una tierra pantanosa que dificultaba enormemente su tránsito a aquéllos que no conocieran los caminos de acceso. Este lodazar cubierto por espesos cañizales y juncales ofrecía una seria barrera a visitantes hostiles.

Todo el sistema de defensa descrito, tanto artificial como natural, se complementaba con la cooperación desde altos bastiones naturales ocupados por poblados afines al Cerro de los Encaños. Hemos localizado no menos de una docena de estos asentamientos con similares características culturales a las de Villar del Horno, que ocupaban las alturas más defendidas del valle, de tal manera situados que, desde cualquiera de ellos pueden verse los más próximos. Es lógico pensar ante lo descrito anteriormente que estas gentes pusieron en práctica un 


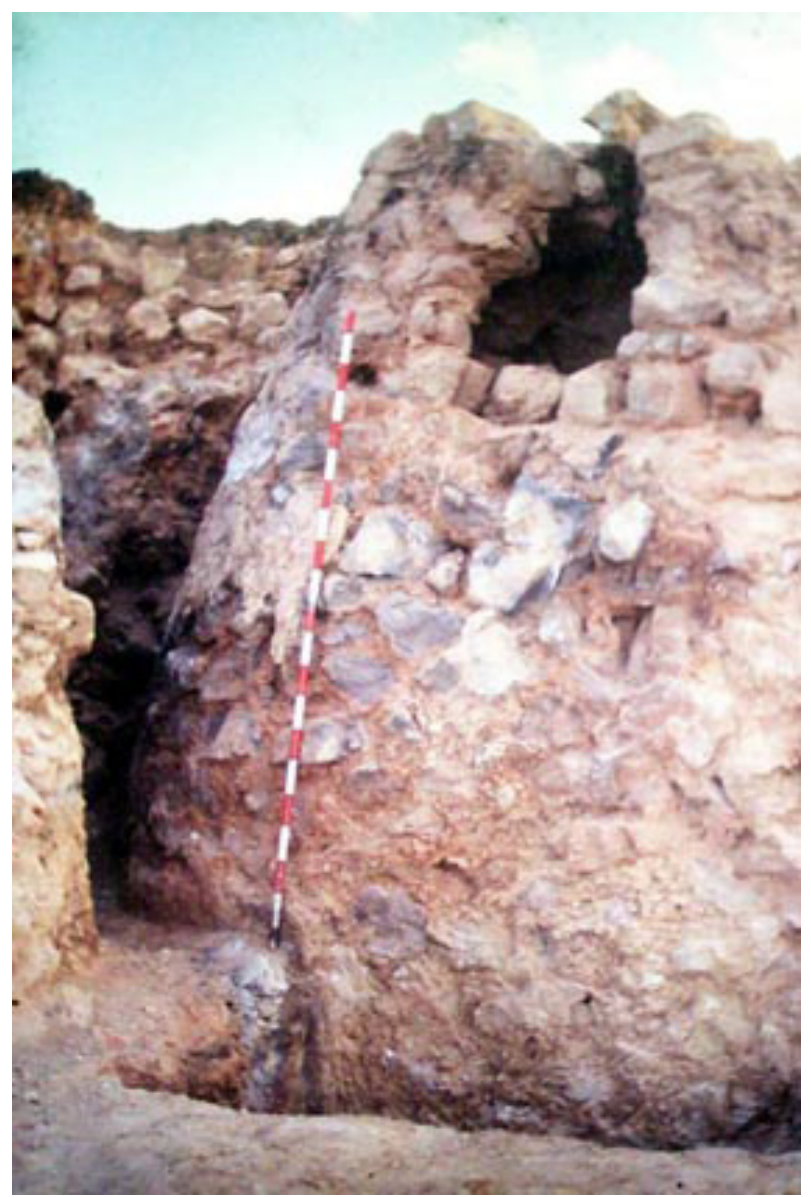

Figura 6-. Bastión defensivo del yacimiento de Castillejo II en Naharros, próximo al Villar del Horno.

sistema de comunicación visual y acústico suficientemente eficaz para garantizar la llegada de un mensaje a su destino, evitando de esta manera un ataque sorpresivo (Gómez Ruíz, 1999, 83-87).

A pesar de todo lo expuesto, el Cerro de los Encaños sucumbió (y, posiblemente, todos los poblados de la zona) ante fuerzas muy superiores.

Villar I fue destruido violentamente y el cerro quedó deshabitado. Posteriormente, fue de nuevo ocupado por gentes con las mismas tradiciones, técnicas alfareras y actividades económicas. Las construcciones de Villar II se superpusieron a las del primer poblado, beneficiándose sus habitantes de una mayor actividad comercial desarrollada por gentes ajenas a la zona. Este segundo asentamiento corrió la misma suerte que el primero, pues se abandonó al final del siglo IV o principios del siglo III a. d. J. C.

Atestiguado por algunas cerámicas griegas en el yacimiento y cerámicas estampilladas. Villar II fue también violentamente destruido, quedando el
Cerro de los Encaños por mucho tiempo despoblado (Gómez Ruiz, 1999, 83-87).

Una pobre y reducida población, siete u ocho siglos más tarde, consideró el lugar idóneo para establecerse. Algunos fragmentos de Sigillata Africana, unas cuantas tejas y diversos pozos o basureros rellenos de cenizas, testimonian una temporal presencia de nuevos habitantes; pero tras el abandono de éstos, que dejaron pocos vestigios de su presencia, ningún otro grupo humano consideró al Cerro de los Encaños zona apropiada para alzar sus viviendas.

\section{MATERIAL RECUPERADO}

Para deducir la vida cotidiana en el Cerro de los Encaños vamos a enumerar y describir muy someramente algunos de los materiales recuperados en sucesivas campañas. En primer lugar, la cerámica (Urbina Martínez, 2015), el material más abundante abandonado en el poblado tras su destrucción. La mayoría de las veces aparece in situ y su abundancia está justificada por su menor valor económico en relación a otros materiales, tales como metales, armas, herramientas, etc., que serían los útiles con más interés transportados por los habitantes en su huida o los más buscados en el expolio tras la conquista del cerro (Charro y Cabrera, 2011).

Las cerámicas del Cerro de los Encaños fueron fabricadas a mano y a torno (Mena Muñoz, 1984). Las dos técnicas están representadas en ambos poblados, pero en Villar I es porcentualmente más abundante la cerámica a mano; fenómeno que se invierte en Villar II. Ello nos indica una influencia comercial más pronunciada en el más reciente poblamiento, Villar II, considerando la cerámica a torno procedente de otras zonas y, autóctona, la cerámica a mano, y con ello la iberización de la región (Cabello Caja, 1992). Pero para mejor comprensión de lo dicho, podemos clasificar la cerámica del Cerro de los Encaños en:

a. Cerámica de cocina. Muy quemada por el uso constante y su aproximación al fuego. Ollas, cuencos y vasijas generalmente, de cerámica basta, sin decorar y con desgrasantes gruesos.

b. De almacenaje. Grandes vasijas a mano con vestigios de material orgánico en sus paredes interiores, o tinajas a torno decoradas, procedentes de otras zonas y utilizadas, posiblemente, para el transporte y almacenaje de líquidos y áridos. Son típicas, también, 
para estos menesteres, las urnas de orejetas, representadas en Villar del Horno y tan típicamente abundantes en las necrópolis de la época como urnas funerarias. Sospechamos que serían los recipientes adecuados para contener productos lácteos, miel y alguna que otra bebida alcohólica.

c. De uso doméstico. Utilizadas como vajilla para la comida en común: cuencos, vasos, copas, vasijas y platos a mano, con o sin decoración pero siempre cuidada su superficie, y a torno, generalmente decorada o con engobe. Una cerámica especial, procedente al principio de los núcleos del Levante o del S, aparecen en Villar I y es relativamente abundante en Villar II. Nos referimos a la cerámica de pasta gris.

d. De lujo. Representada por la cerámica a mano pintada, tipo Carrascosa, o de retícula bruñida, tipo Cástulo, en Villar I; y por diminutos vasos carenados de cerámica gris, platos de reducido diámetro o copas áticas de barniz negro de finales del siglo $\mathrm{V}$ o principios del IV, que creemos de uso ritual o funerario. Estas copas áticas de Villar II, son similares a las aparecidas en la tumba 25 de la necrópolis de Los Villares, provincia de Albacete (Blánquez Pérez, 1980, 37-45).

e. Aunque de cerámica a mano, podemos incluir en este apartado un pebetero de uso ritual hallado en Villar II. (Fig. 7). Ya apareció un fragmento de pebetero en Reillo, aunque de cerámica fabricada a torno y decorada con una serpiente, como testimonio de la existencia de un culto ofiolátrico. También se ha evidenciado la práctica de sacrificios rituales de animales domésticos en el Cerro de la Mesa (Cabrera Díaz et al., 2014).

f. En cuanto al ritual religioso contamos hoy en día con la evidencia del templo o santuario en el yacimiento de La Cava, en Garcinarro, en el valle de Eltomira en Cuenca (Valero Tévar, 2016).

g. Plataformas de arcilla que, según Ortega Blanco (Ortega y del Valle, 2004,175-185), podrían tratarse de altares domésticos, como uno similar aparecido en Villar del Horno, pero con la singularidad que este último cubría seis vasos de cerámica colocados intencionadamente bajo la placa circuloide de arcilla.
De hecho, los pueblos prerromanos que ocuparon la Península Ibérica (Caro Baroja, 1976) manifestaban el mismo gusto por el material de importación, tanto cerámico como metálico, que el desarrollo comercial proporcionó a lo largo de todo el territorio; comunicando la zona tartésica con el área oriental (Almagro et al., 2006), y el mundo céltico con el ibérico de la franja mediterránea.

\section{Metales}

La muestra más representativa es el bronce; con remaches, argollas, paredes de vasos, agujas y, sobre todo, fíbulas de distintos tipos. Le sigue el hierro, escaso en todo el yacimiento salvo el depósito hallado en el recinto 14 , al que haremos referencia, por su importancia, más adelante (Lorrio, 2007, 251 y 278).

Un pequeño alambre de plata doblado en forma de grapa, que ya fue publicado, apareció en un nivel de Villar I (Gómez Ruiz, 1986, 332).

Una de las últimas campañas proporcionó un disco de plomo, posiblemente un ponderal o medida de peso.

\section{ELEMENTOS DE TRABAJO}

Están representados en el Cerro de los Encaños los hornos de cocer, de adobe o masa arcillosa, en forma de colmena, sobre base o plataforma, también de adobes. Su uso principal sería la cocción del pan y otros menesteres culinarios, aunque no descartamos una esporádica finalidad para la cocción de cerámica a mano.

Han aparecido en el poblado algunos molinos barquiformes, tanto en el primer nivel como en el segundo. A veces, inutilizados éstos por rotura, se han aprovechado para la construcción (Ortega y del Valle, 2004).

La abundancia de pondera y fusayolas nos indica el cotidiano trabajo textil por parte de la población femenina. Algunos huesos trabajados para enmangues de cuchillos $\mathrm{u}$ objetos punzantes nos hace intuir una actividad artesana y hogareña. La aparición de algunos clavos de hierro nos testimonia el trabajo de la madera.

La aparición de algunos alisadores líticos (mal llamados pequeñas hachas votivas por su parecido con estos útiles) nos indica el trabajo del pulimento de la madera y el hueso y el curtido de pieles (Urbina Martínez, 2015).

\section{ADORNOS PERSONALES}

Una prospección anterior a nuestras excavaciones realizada por D. Francisco Suay (Almagro Gor- 

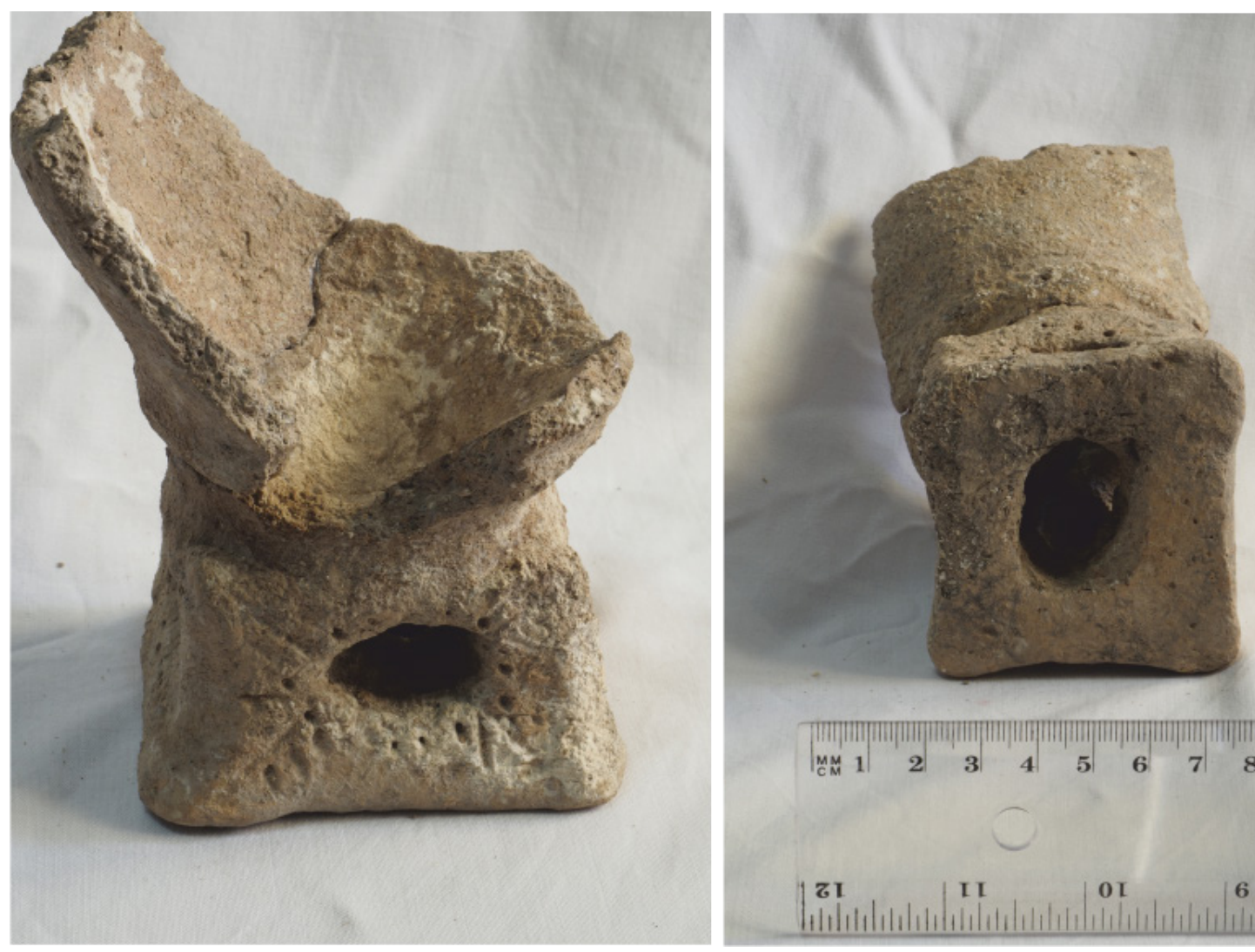

Figura 7.- Pebeteros.

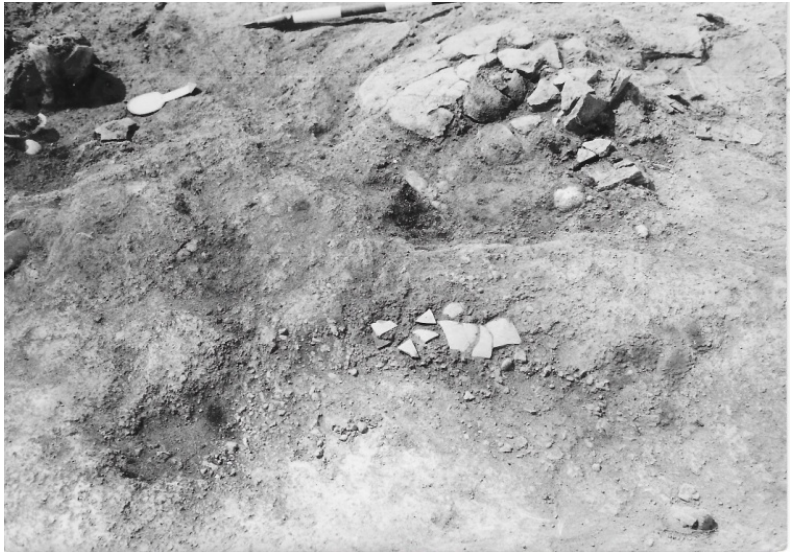

Figura 8.- Plataforma de arcilla fragmentada por desplome y cubriendo seis vasos de cerámica a mano.
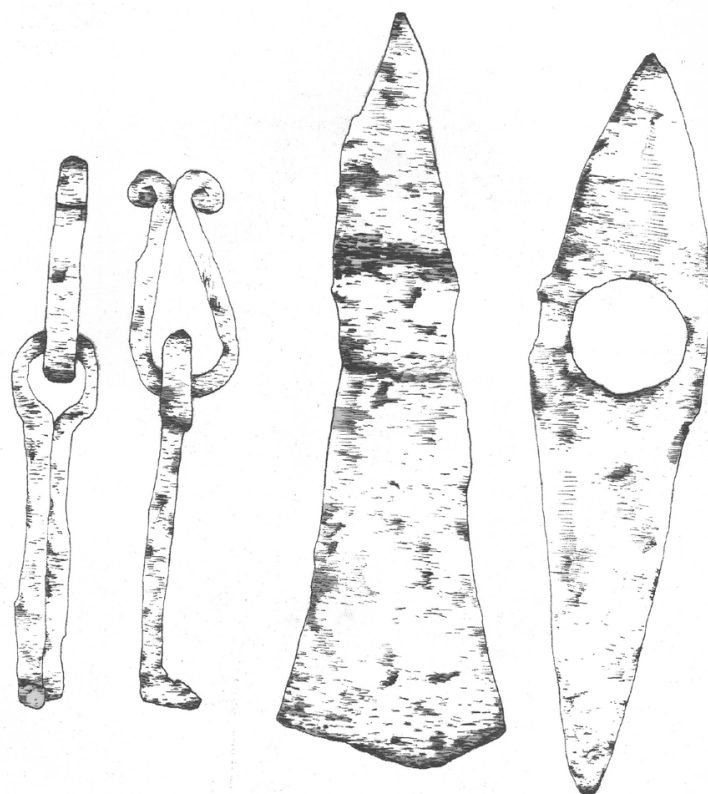

Figura 9.- Asidero de hierro y hachuela 

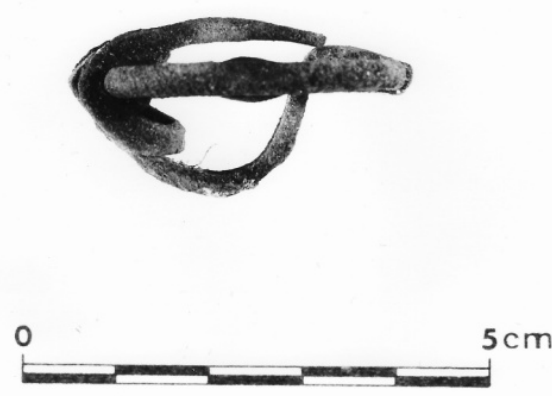

Figura 10.- Fíbula hispánica de bronce

bea, 1978, 112) proporcionó un broche de cinturón de un solo garfio con un grifo grabado. Un broche similar se halló en la tumba de la cuadrícula 5 de la necrópolis de Los Villares en Albacete (Blánquez Pérez, 1980, 37-45).

Relativamente abundantes son las fíbulas, en bronce unas y en hierro otras, y de diferentes tipos. Aretes, agujas y, posiblemente eslabones de cadenas para el cuello son otros tantos objetos de bronce aparecidos en el poblado y en otros similares.

Con material lítico se fabricaron cuentas de collar de pasta vítrea (procedentes del exterior) y una pizarra trabajada, posiblemente, como paleta de afeites (Gómez Ruiz, 1986, 332).

Se halló, también, un colgante de concha marina, trabajado y horadado, similar a uno aparecido en la necrópolis de Las Madrigueras en Carrascosa del Campo (Almagro Gorbea, 1969) y otro en Riba de Saelices en Guadalajara (Cuadrado, 1968).

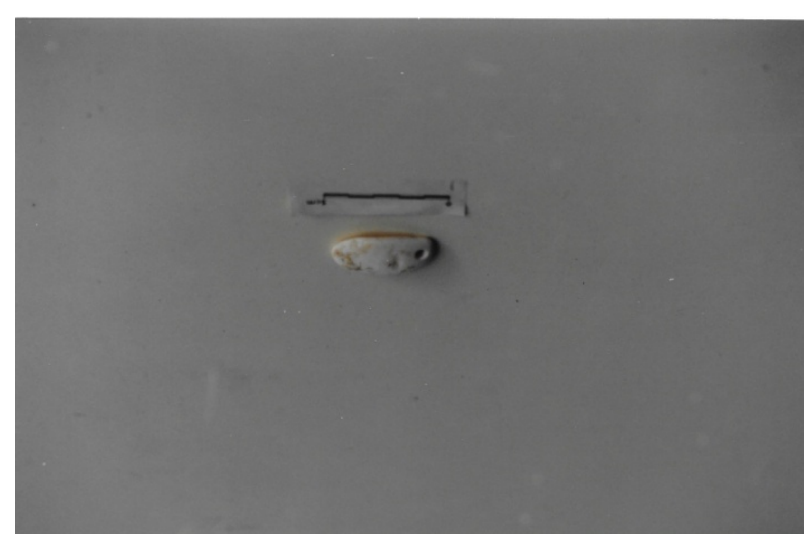

Figura11.- Colgante de concha marina horadado.
Por último, y formando parte del depósito de hierros aparecidos en el Recinto-14, citaremos una pinza de depilar de hierro, muy similar (aunque sin decoración) a la aparecida en la necrópolis ibérica de Los Torviscales (Córdoba). Pero los paralelos más próximos se hallan en Buenache de Alarcón. Aparece una pinza de bronce en la tumba 3; otras dos, en la incineración 9; otra de hierro, en la incineración $\mathrm{n}^{\mathrm{o}} 11$ y otras dos del mismo metal en la incineración 14 (Losada Gómez, 1966, 36).

\section{Material LÚdico INFANTIL}

Hemos de deducir que los niños del Cerro de los Encaños disfrutaron de un corto periodo de tiempo dedicado exclusivamente al juego. Tenderían a emular las actividades laborales de los mayores, y cuando la edad se consideraba suficiente, se irían incorporando al mundo del trabajo, por necesidad perentoria de todas las comunidades dedicadas a actividades agropecuarias. ( Ruiz y Álvarez, 2015)).

Sólo una clase de objetos recuperados podemos asociarlos al juego infantil (y, posiblemente, al de los adultos); se tratan de unas bolas de barro cocido de irregular factura y un diámetro que oscila entre los $2^{\prime} 5 \mathrm{~cm}$ y los $3 \mathrm{~cm}$. Considerando que son juguetes de niños, de fácil fabricación, y también de frecuente fractura (de ahí su irregularidad e imperfección), podemos equipararlas a los actuales boliches o canicas. En Villar han aparecido seis de estas bolas, pero son relativamente frecuentes en otros yacimientos, tales como en la necrópolis de Carabias (donde aparecen 18 de estas bolas) y la necrópolis de la Olmeda; en Riba de Saelices aparecen 50 de estos objetos entre varias tumbas. Las tres necrópolis anteriores se hallan en la provincia de Guadalajara. También aparecen en el poblado de La Hoya (Álava), realizadas en piedra, unas, y en barro cocido, otras; aunque aquí se interpretan, creemos que desacertadamente, como elementos de contabilidad o señales de cambio en un momento premonetal (Llanos, 1980, 6-12), así como en Sepúlveda (Segovia), con 19 ejemplares, tanto en piedra como en cerámica, lisas o decoradas (Blanco García, 1998, 137-174).

\section{EL DEPÓSITO DE HIERROS DEL RECINTO-14}

Las distintas campañas de excavaciones arqueológicas en el cerro de los Encaños se han caracterizado por la relativa escasez de material metálico recuperado, sólo representado por pequeños objetos y frag- 


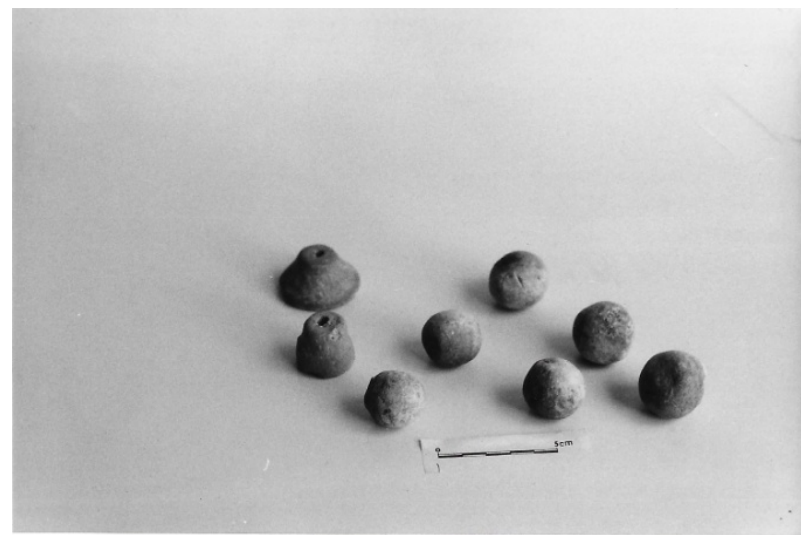

Figura 12.- Bolas aparecidas en el yacimiento junto a dos fusayolas.

mentos de ellos, tales como aretes, fíbulas, alambres, clavos, plaquitas, etc. Creemos que ello puede deberse a dos causas (posiblemente, complementarias). Por su gran valor económico, serían los primeros materiales rescatados en una precipitada huida. Por otra parte, los invasores, vencido todo tipo de resistencia, se dedicarían al expolio del poblado y a su posterior incendio (Sierra Delage, 2002).

Casualmente, dos recintos de los excavados en la actualidad no fueron totalmente expoliados, bien por ocultamiento de los materiales bajo la capa de cenizas, bien por desorientación de los expoliadores. En el recinto-11 hallamos un telar quemado in situ, y sobre la mancha de carbones y ceniza, los siete pondera correspondientes a la estructura de madera y fibra textil. Se conservaba parte del pavimento donde aparecieron algunas fusayolas y, en el centro, un hogar limitado por adobes en posición vertical; al lado del hogar, in situ y completo, un plato de cerámica gris (Mena Muñoz, 1984).

El Recinto-14, al S del anterior y contiguo a éste, presenta un tabique de adobes caídos sobre el pavimento, y ello, posiblemente, "selló" el depósito de utillajes de hierro. Bajo el tabique de adobes caído, un segundo telar calcinado con siete pondera entre sus cenizas; sobre el pavimento cerámicas de diversos tipos representados en el yacimiento, algunas fusayolas y pequeños utillajes de bronce como fíbulas, plaquitas y algunos remaches (Ortega y del Valle, 2004). En el centro del recinto, sobre el pavimento y bajo una hilada de adobes se hallaron diversos aperos de labranza y otras herramientas de hierro entre las que destacan: un legón, una hachuela, una alcotana, un podón, unas tijeras, la hoja de un cuchillo, un pico, un punzón, una pieza de hier-

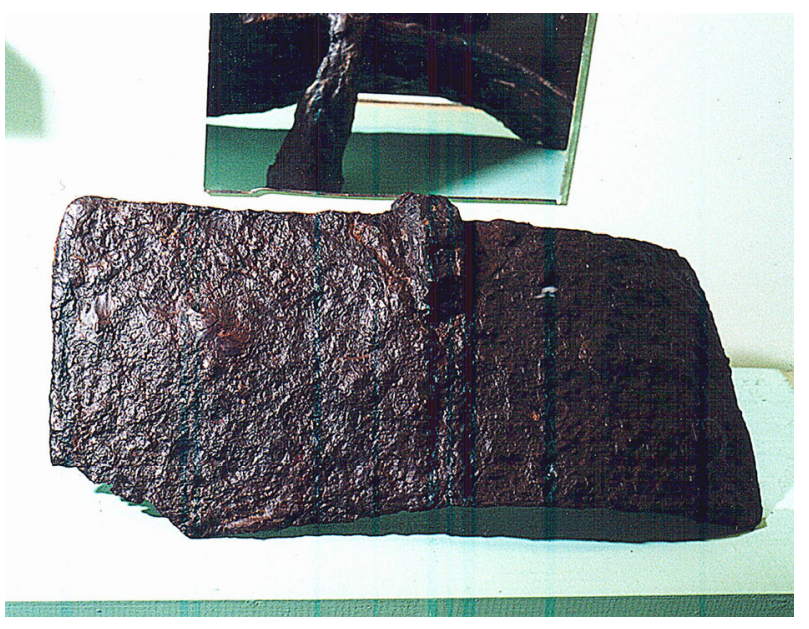

Figura 13-. Azadón de grandes dimensiones

ro ensamblada, posiblemente, un gozne de puerta, una fíbula de hierro, algunos clavos, una argolla de hierro y parte de otras, algunos remaches, una grapa de hierro, una pinza de depilar, una fíbula de bronce de resorte, algunas laminillas de bronce con remaches del mismo material, dos piezas de hierros enlazadas, posiblemente, un tirador de puerta o un asa de arca, la reja de un arado y parte del herraje del mismo (Jimeno et al.,1999).

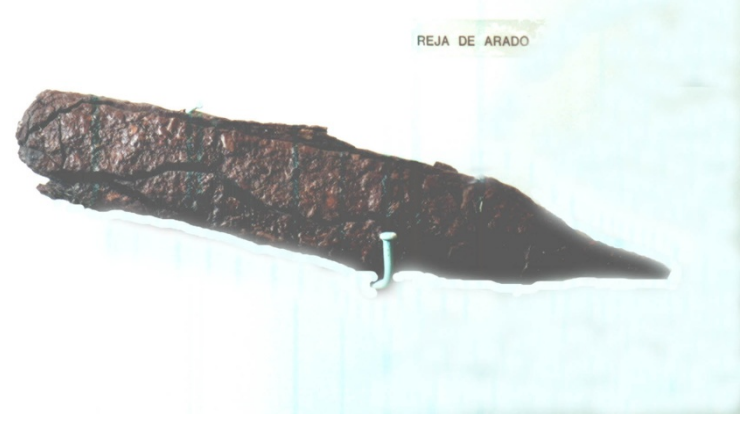

Figura 14.- Reja de arado aparecida en el recinto 14.

Algunos de estos materiales se han restaurado y hoy se exponen en el Museo Provincial de Cuenca; otro grupo de objetos se ha consolidado y el resto quedó depositado en los almacenes del museo por falta de partidas económicas para su tratamiento.

Describiremos los más característicos:

a. Legón o gran azadón, restaurado; presenta unas proporciones de $35 \times 16 \mathrm{~cm}$. El paralelo más cercano lo hallamos en Numancia, donde aparece la mitad de un ejemplar muy 
parecido (Jimeno et al., 1999).

b. Hachuela de doble filo, apta para el talado. Se conservan cinco ejemplares procedentes de Numancia (Jimeno et al., 1999)

c. Hacha-pico; también aparecen varias en $\mathrm{Nu}-$ mancia (Jimeno et al., 1999), pero existen otros paralelos más antiguos, en Soto de $\mathrm{Me}$ dinilla (Valladolid). En el Museo Regional de Lagos (Portugal) se exponen dos herramientas similares con números de inventario 202 y 203 respectivamente, con una longitud de $25 \mathrm{~cm}$ el primero y $20 \mathrm{~cm}$ el segundo. Proceden de Vale da Maia, Arrifos do Poço (Aljezur).

d. Podón. Fue restaurado y se conserva en el Museo Provincial de Cuenca. Aparecen dos ejemplares similares en Numancia (Jimeno et al., 1999)

e. Tijeras. En el mismo depósito aparecieron las dos hojas de unas tijeras. Estas herramientas son frecuentes en los yacimientos del Hierro, por lo que suponemos un uso muy generalizado. El paralelo más cercano lo tenemos en la necrópolis de Buenache de Alarcón en Cuenca (Losada Gómez, 1966, 38), pero también han aparecido en el yacimiento de Los Torviscales (Córdoba), en La Hoya (Álava) y, por supuesto, en Numancia, donde se conservan 27 ejemplares (Jimeno et al., 1999)

Forma parte de este depósito una hoja de cuchillo que, sin restaurar, se conserva en los almacenes del Museo Provincial con otros objetos del mismo yacimiento. Los cuchillos (algunos de ellos conservando su mango de hueso) son frecuentes en los yacimientos del Hierro. Concretamente, en $\mathrm{Nu}-$ mancia han aparecido 60 ejemplares (Jimeno et al., 1999)

Apareció también en este lugar un conglomerado de hierro que ha quedado depositado en los almacenes del museo. Aún sin tratar ni limpiar, podemos identificarlo con un gozne o bisagra de grandes proporciones.

Una pieza de hierro de forma cónica alargada, hueca y de sección circular, parecida a un regatón; pero no queremos identificarlo de esta manera ante la carencia total de armas en el poblado y las características agrícolas de casi todo el utillaje del depósito. Pensamos, más bien, en un punzón de hierro de utilidad diversa para enmangar sobre madera.
Algunos remaches de hierro que podríamos asociar al gozne anteriormente mencionado.

Una grapa de hierro de $55 \mathrm{~cm}$ de longitud, posiblemente asociada a los anteriores remaches. Grapas como ésta aparecen en Numancia, 32 en total (Jimeno et al., 1999); más próximo al yacimiento encontramos varias grapas de hierro en los ajuares de la necrópolis de Olmedilla de Alarcón en Cuenca (Almagro Gorbea, 1978) y en el poblado de Illescas en Toledo, de donde se recogieron seis ejemplares (Valiente y Balmaseda, 1979, 153-210).

Una argolla de hierro y partes de otras que podríamos asociar a bocados de caballo, pero que por estar tan generalizada su utilidad y no haber aparecido en el depósito otras partes de dicho utillaje, citamos aquí su posible uso con las suficientes reservas.

Pinza de depilar. Conservada en los almacenes del museo. Pieza consolidada solamente, por lo que no puede atisbarse ninguna clase de decoración. Perdido de antiguo el alambre o argolla para colgarla, presenta unas dimensiones de 9'5 $\mathrm{cm}$ de longitud por $2 \mathrm{~cm}$ de anchura en su extremo suelto. Los paralelos más próximos, como hemos indicado antes, son las numerosas pinzas aparecidas en la necrópolis de Buenache de Alarcón (Losada Gómez, 1966), pero el ejemplar más parecido se ha hallado en Los Torviscales en Córdoba (Vaquerizo Gil, 1986).

Reja de arado. Restaurada y expuesta en una de las vitrinas del Museo Provincial; es, posiblemente, el ejemplar más antiguo conocido de este periodo en la Península, si aceptamos la cronología propuesta para este poblado; extendidas ampliamente estas rejas de arados por todo el territorio peninsular a partir de los siglos IV y III, y su abundante representación numismática en los siglos II y I a.d. J. C., nos demuestra su uso generalizado en época prerromana (Barril Vicente, 1999).

Herrajes correspondientes al arado. El material lignoso ha desaparecido, quizá, por combustión; se conservan algunos elementos férricos del arado.

Una doble pieza enlazada de hierro. Una de las partes, remachada para sujetar por el interior de una puerta o tapa de baúl. La otra, movible y con función de asidero, rematada en dos artísticas volutas (Lám. 9).

Una fíbula de bronce, otra de hierro, algunos clavos de distinta longitud, una laminilla de bronce con remaches y algunos conglomerados de hierro sin identificar formaron este singular depósito metálico en uno de los recintos del poblado. 


\section{CONCLUSIONES}

Los pueblos que se establecieron durante el siglo VII a.C. en el alto curso del Cigüela explotaron agropecuariamente el amplio y fértil valle por donde discurre el río.

Conscientes de vivir una época de gran inestabilidad política y social, basaron su defensa en la colaboración de poblados afines situados alternativamente en los bordes del valle y estratégicamente ubicados para que un mensaje visual o acústico enviado por uno de dichos poblados sería en poco tiempo receptado por todos los habitantes de la zona.

Por el material proporcionado en varias campañas de excavaciones arqueológicas en el Cerro de los Encaños deducimos la existencia de frecuentes y regulares relaciones comerciales y culturales con pueblos procedentes del este peninsular en períodos de estabilidad, y bajo la permanente vigilancia de todos los núcleos poblacionales fuertemente fortificados (Almagro Gorbea, 1987).

En un momento dado a finales del siglo III a. C, gente foránea consiguieron eliminar bruscamente el sistema social y económico de esta comunidad de agricultores y ganaderos que explotaron con éxito y durante largo tiempo un área que estimamos en unos 50-70 kilómetros cuadrados.

\section{BiBLIOGRAFÍA}

Almagro Gorbea, M. (1969), La necrópolis de Las Madrigueras. Carrascosa del Campo (Cuenca), Excavaciones Arqueológicas en España, 41, Madrid.

Almagro Gorbea, M. (1978), "La iberización de las zonas orientales de La Meseta", Ampurias, $3840,93156$.

Almagro Gorbea, M. (1988), "La cultura de la Edad del Bronce y la Edad del Hierro en Castilla LaMancha”, I Congreso de Historia de CastillaLa Mancha, II, Toledo, 166180.

Almagro Gorbea, M., Jiménez Ávila, J., Lorrio, A.L., Mederos Martín, A. y Torres, M. (2006), La necrópolis de Medellín I. La excavación y sus hallazgos, Bibliotheca Archaeologica Hispana, 26, Real Academia de la Historia, Madrid.

Barril Vicente, M. (1999), "Arados prerromanos de la Península Ibérica: las rejas y su distribución zonal en el interior peninsular”, IV Simposio sobre los Iberos, Zaragoza, 89101.

Blanco García, J. F. (1998), "La Edad del Hierro en Sepúlveda (Segovia)”, Zephyrus, LI, 137174.

Blánquez Pérez, J. J. (1980), "Túmulos ibéricos: necrópolis de Los Villares", Revista de Arqueología, 36,3745 .

Cabello Caja, R. (1992), "La cerámica pintada de la II edad del Hierro en la cuenca del media del Tajo", Norba, 1112, 98128.

Caro Baroja, J. (1976), Los pueblos de España, Madrid.

Contreras Martínez, M., Märtens Alfaro, G., Ruiz Zapatero, G. y Baquediano, E. (2014), "Oppidum, urbanismo y organización de los espacios de hábitat en El Llano de la Horca (Santorcaz. Madrid)", Zona Arqueológica, 7, 111124

Charro Lobato, C. y Cabrera Díez, A. (2011), "El yacimiento arqueológico del Cerro de la Mesa y su entorno (Alcolea del Tajo, Toledo), Los vínculos entre el hábitat y el paisaje (J.P. López, D. Hernández y J. García, Coords.), Madrid, 207216.

Cuadrado, E. (1968), Excavaciones en la necrópolis celtibérica de Riba de Saelices (Guadalajara), Excavaciones Arqueológicas En España, 60, Madrid.

García Bellido, A. (1968), España y los españoles hace dos mil años según la Geografía de Strabón, Madrid.

García Huerta, R.,Morales, F.J; Vélez, J, Soria, L. y Rodríguez, D. (2006), "Hornos de pan en la Oretania Septentrional”, Trabajos de Prehistoria, 63/1, 157166 .

Gómez Ruiz, A. (1986), El Cerro de los Encaños. (Villar del Horno. Cuenca), Noticiario Arqueológico hispano, 27, 265350)

Gómez Ruiz, A. (1999), "La Edad del Hierro en el alto curso del río Cigüela", I Jornadas sobre el patrimonio de La Manchuela conquense, Cuenca, 8387.

Gras, E., Mena, P. y Velasco, F. (1984), "La ciudad de Fosos de Bayona (Cuenca)", Revista de Arqueología, 36, 4857.

Jimeno, A., De la Torre, J. I., Berzosa, R. y Granda, R. (1999), "El utillaje de hierro en Numancia y su información económica”, IV Simposio sobre los Iberos (F. Murillo Bozota, Coord.), Zarago$\mathrm{za}, 103113$.

Llanos, A. (1980), "El poblado prerromano de La Hoya", Revista de Arqueología, 10, 712.

Lorrio, A.J. (2007), "Historiografía y nuevas inter- 
pretaciones: la necrópolis de la Edad del Hierro de Haza de Arca (Uclés, Cuenca)", Cesaraugusta, 78, 251275.

Losada Gómez, H. (1966), La necrópolis de la Edad del Hierro en Buenache de Alarcón (Cuenca), Trabajos de Prehistoria, XX, Madrid.

Maluquer de Motes, J. (1979), Los pueblos ibéri$\cos$, Historia de España dirigida por Menéndez Pidal, 1/3., Madrid, 305370.

Martín Valls, R. (1997), "La Segunda Edad del Hierro: consideraciones sobre su periodización”, $Z e$ phirus, 3940, 5986.

Mena Muñoz, P. (1984), Catálogo de cerámicas de necrópolis de la Edad del Hierro. Museo de Cuenca, Boletín del Museo Provincial de Cuenca, I, Madrid.

Ortega Blanco, J. y del Valle Gutiérrez, M. (2004), "El poblado de la Edad del Hierro del Cerro de la Mesa. Primeros resultados (Alcolea del Tajo, Toledo)", Trabajos de Prehistoria, 61/1, 175185.

Rodríguez Hernández, J. (2018), Las comunidades de la Edad del Hierro en el occidente de La Meseta: cultura material, poder y sociedad, Universidad Complutense, Madrid.

Ruiz Zapatero G. y Álvarez Sanchiz, J. R. (2015), "¿Centros de poder? Sociedad y poblamiento en La Meseta Norte española (ca 800500 a.C.)”, Vegueta. Anuario de la Facultad de Geografía e Historia, 15, 211233.

Sierra Delage, M. (2002), El yacimiento ibérico Fuente de la Mota (Barchín del Hoyo, Cuen$c a$ ), Diputación Provincial de Cuenca, Cuenca.

Urbina Martínez, D. (2015), "Un asentamiento de la Edad del Hierro en Torrigue. Noblejas (Toledo)”, Cuadernos de Prehistoria y Arqueología de la Universidad Autónoma de Madrid, $41,5568$.

Valero Tévar, M.A. (2016), Paisaje alcarreño en el Valle de Altomira (Uclés), Cuenca.

Valiente Cánovas, S. (1981), "Pico de la Muela (Valera de Abajo. Cuenca)", Noticiario Arqueológico Hispano, 12, 97254.

Valiente Cánovas, S. (1982), "Excavación en el poblado de Bonilla (Cuenca)", Noticiario Arqueológico Hispánico, 14, 121134.

Valiente Cánovas, S. (1986), "La cultura del la Edad del Hierro", 130 años de Arqueología Madrileña, Real Academia de Bellas Artes de San Fernando, Madrid, 153210.

Valiente Cánovas, S. y Balmaseda Muncharaz, L.J. (1979), "Excavación en El Cerrón (Illescas, To- ledo)”, Noticiario Arqueológico Hispano, 7, 153210.

Vaquerizo Ruiz, D. (1986), "Pinza de depilar en la necrópolis ibérica de Los Torviscales”, Revista de Arqueología, 66, 4992. 
